Saudi Journal of Oral and Dental Research

Abbreviated Key Title: Saudi J Oral Dent Res

ISSN 2518-1300 (Print) |ISSN 2518-1297 (Online)

Scholars Middle East Publishers, Dubai, United Arab Emirates

Journal homepage: https://saudijournals.com/sjodr

Case Report

\title{
Fibrous Dysplasia: Case Report \& Review
}

\author{
Dr. Bilal Afzal Amir ${ }^{*}$, Dr. Jennie Clarence ${ }^{2}$, Dr. Anam Raza ${ }^{3}$, Dr. Pooja Agroya ${ }^{4}$, Dr. Archana Agroya ${ }^{5}$, Dr. Heena \\ Tiwari $^{6}$ \\ ${ }^{1}$ PG Student, Depatrment of Prosthodontics Crown and Bridge and Implantology, Sri Sai College Of Dental Surgery, Vikarabad, India \\ ${ }^{2}$ Consultant Oral and Maxillofacial Surgeon, Grace Dental Clinic, 5G, Daud Nagar, Mevala Ki Bagiya, Naini, Prayagraj, Uttar Pradesh 211008, India \\ ${ }^{3}$ BDS, MS, Baqai Medical University, Pakistan, University of Denver-CO, USA \\ ${ }^{4} \mathrm{MDS}$, Senior Lecturer, Department of Prosthodontics, Crown \& Bridge, Sri Sai College of Dental Surgery, Vikarabad, India \\ ${ }^{5}$ MDS, Senior Lecturer, Department of Periodontology \& Implantology, Sri Sai College of Dental Surgery, Vikarabad, India \\ ${ }^{6}$ BDS, PGDHHM, Ex-Government Dental Surgeon, Kondagaon, Chhattisgarh, India
}

DOI: $10.36348 /$ sjodr.2020.v05i09.007 $\quad$ Received: 22.08.2020 /Accepted: 30.08.2020 |Published: 03.09 .2020

*Corresponding author: Dr. Bilal Afzal Amir

\section{Abstract}

Fibrous dysplasia (FD) is a non-neoplastic developmental hamartomatous disease of the bone, characterised by a blend of fibrous and osseous elements in the region. It is a lesion of unknown etiology, uncertain pathogenesis, and diverse histopathology. With an incidence of 1:4000-1:10,000 it seems to be a rare disease. It represents approximately $2.5 \%$ of all bone lesions and about $7 \%$ of all benign bone tumors. The objective of this paper is to present an unusual case of Fibrous Dysplasia. A patient with chief complaint of pain and swelling in right side of the mandible was examined. OPG showed poorly defined mixed density pattern. Ultrasound of thyroid revealed enlarged nodes. CBCT revealed hypodense area. CT also showed features of osteomyelitis. Bone scan suggested fibrous dysplasia, which was confirmed by biopsy. Patient also had deficiency of Vitamin D.

Keywords: Fibrous dysplasia, Monostotic, Polyostotic, Zoledronic acid, Cholecalciferol.

Copyright @ 2020: This is an open-access article distributed under the terms of the Creative Commons Attribution license which permits unrestricted use, distribution, and reproduction in any medium for non-commercial use (NonCommercial, or CC-BY-NC) provided the original author and source are credited.

\section{INTRODUCTION}

Fibrous dysplasia (FD) is a rare bony disorder in which normal bone is replaced by abnormal fibroosseous tissue [2]. There is localized differentiation of the bone-forming mesenchyme affecting a single or many bones, in which skeletal aberrations represent the cardinal feature, but in which certain endocrinopathies, abnormal pigmentation of skin and mucous membrane, and occasionally other abnormalities form part of the entire disease process. The craniofacial skeleton is one of its predilective sites and therefore the temporal bone may become involved. In such instances the disease manifests itself with 1) progressive loss of hearing, 2) increasing obliteration of the external ear canal, and 3) enlargement and distortion of the temporal bone. The first part of this communication is concerned with a review of the clinical and pathological aspects of the disease in general. The second part is concerned with a discussion of the clinical manifestations in the temporal bone, based upon analysis of the entire literature and the authors' personal experience. The indications for surgical intervention include 1) maintenance of a normal outer ear canal, 2) preservation of cochlear and vestibular function, and 3) prevention of secondary complications [2].

\section{CASE PRESENTATION}

A 10 year old patient arrived to us with the chief complaint of intermittent pain in the right lower jaw region since one month. Mild inflammation was observed in the same region without apparent facial deformity. An Orthopantomogram (OPG) revealed a poorly defined mixed density pattern in the right mandibular angle region. Other features like maxilla, mandibular symphysis, parasymphysis, body and ramus of the mandible appeared to be normal. Bilateral glenoid fossae and articular eminences grossly showed no remarkable changes. Bilateral condylar heads also apperared normal. A mixed dentition with multiple erupting teeth having immature root apices was present. Ultrasound of the thyroid gland revealed bilateral subcentimeter intraglandular nodes in submandibular and parotid glands and bilateral enlarged level 2 and 3 nodes. MRI of the mandible showed thickening of the body, ramus and coronoid procecess of the right side of the mandible. Heterogenous T2 signals along with interspersed mild hyperintense signals were noted indicating. Periosteal reaction was also seen along the 
body and amus of the mandible. 3DCT scan of mandible were advised for further investigation of the lesion. 3DCT scan revealed evidence of a poorly defined hypodense area in the right mandibular body region. (Figure $1 \& 2$ ) It showed ill-defined margins extending between the region of the second premolar (tooth number 45) to the second molar (tooth number 47). Evidence of more blastic than lytic changes in the right mandibular region was also seen along with evidence of buccal cortical plate erosion in the same region. Supraperiosteal continuous hyperdense layer was uniformly seen from 45 to angle of mandible which was likely reactive in nature. On the basis of the above mentioned findings, differential diagnoses of 1) acute non-suppurative osteomyelitis of the lower jaw and 2) neoplasm of non-odontogenic origin were made. CT also showed features suggestive of osteomyelitis involving the body, ramus and coronoid processes of the mandible on the right side. A bone scan was advised for further investigation of the lesion. Tc-99m MDP whole body bone scan showed diffuse increased tracer concentration in the ramus and body of the mandible on the right side suggestive of fibrous dysplasia. (Figure 3 \& 4) A biopsy was advised to reach a definitive diagnosis and to rule of any malignant changes. The biopsy of the lesion revealed a gross appearance of multiple soft to bony fragments. Microscopic examination showed fragments of irregular bony trabeculae with no osteoblastic rimming separated by a spindle cell stroma indicative of fibrous dysplsia, thus confirming the diagnosis. Serum Vitamin D test was subsequently advised which revealed deficiency of Vitamin D in the patient. Oral prohylaxis was done to improve the oral hygiene of the patient and the oral cholecalciferol (60000 IU) were prescribed to the patient. Intravenous injections of Zoledronic acid were also administered to reduce bone resorption.

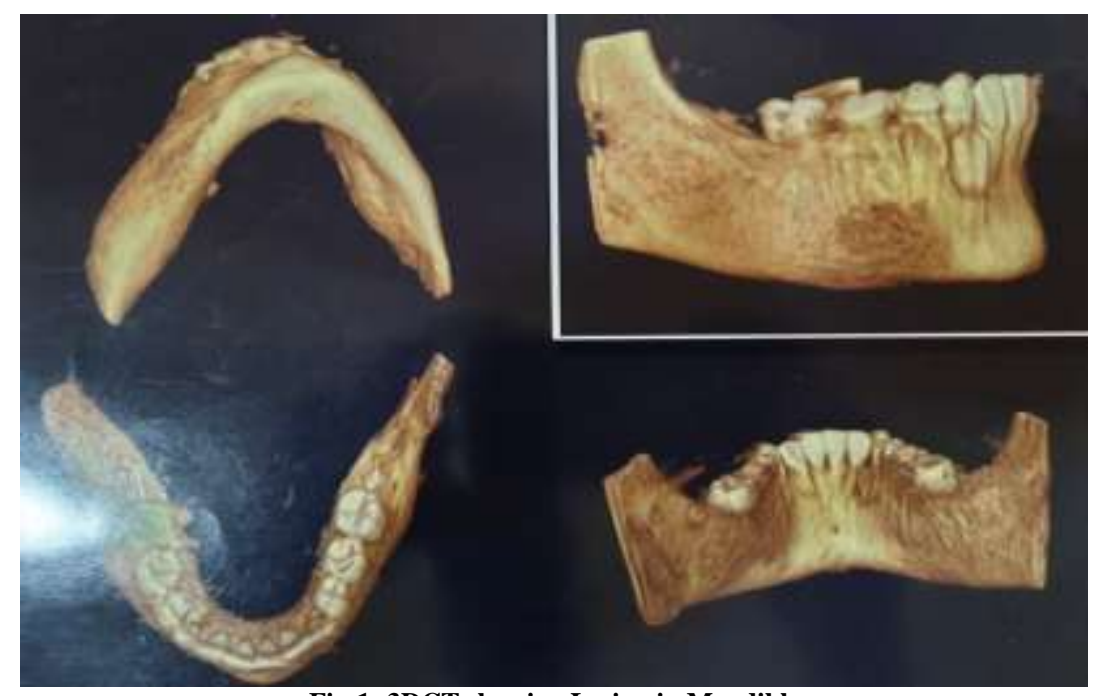

Fig-1: 3DCT showing Lesion in Mandible

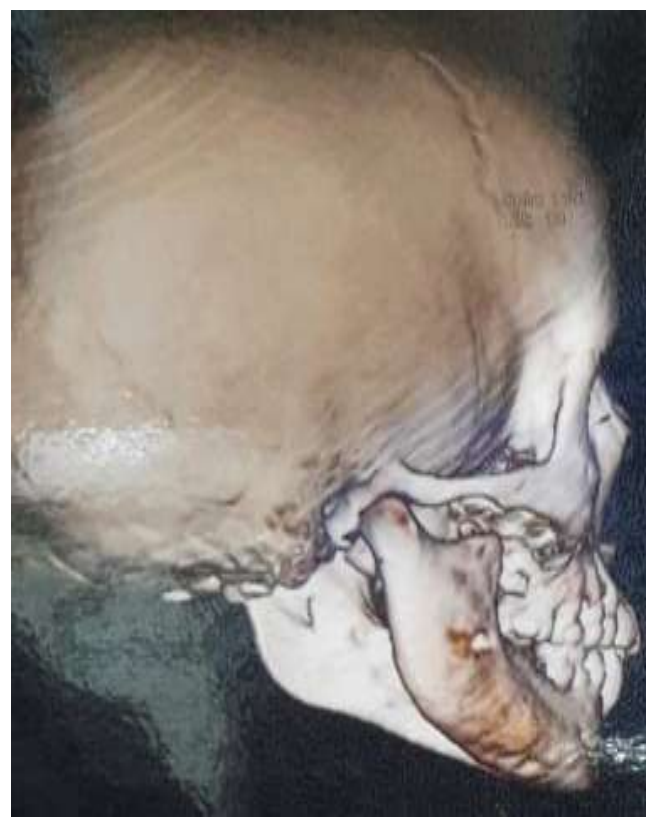

Fig-2: 3DCT showing Lesion in Skull 


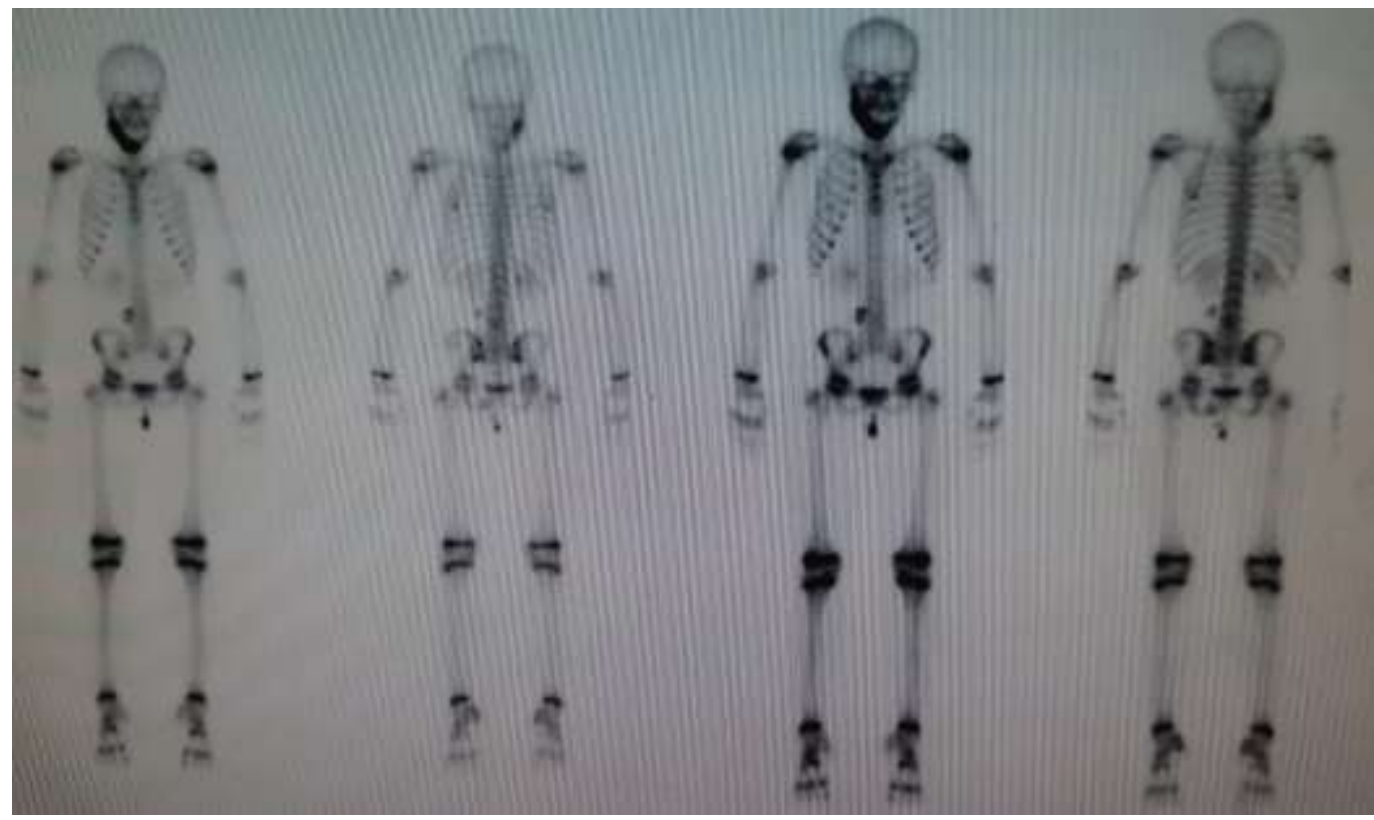

Fig-3: Bone Scintigraphy showing Lesion in Mandible (Plain)

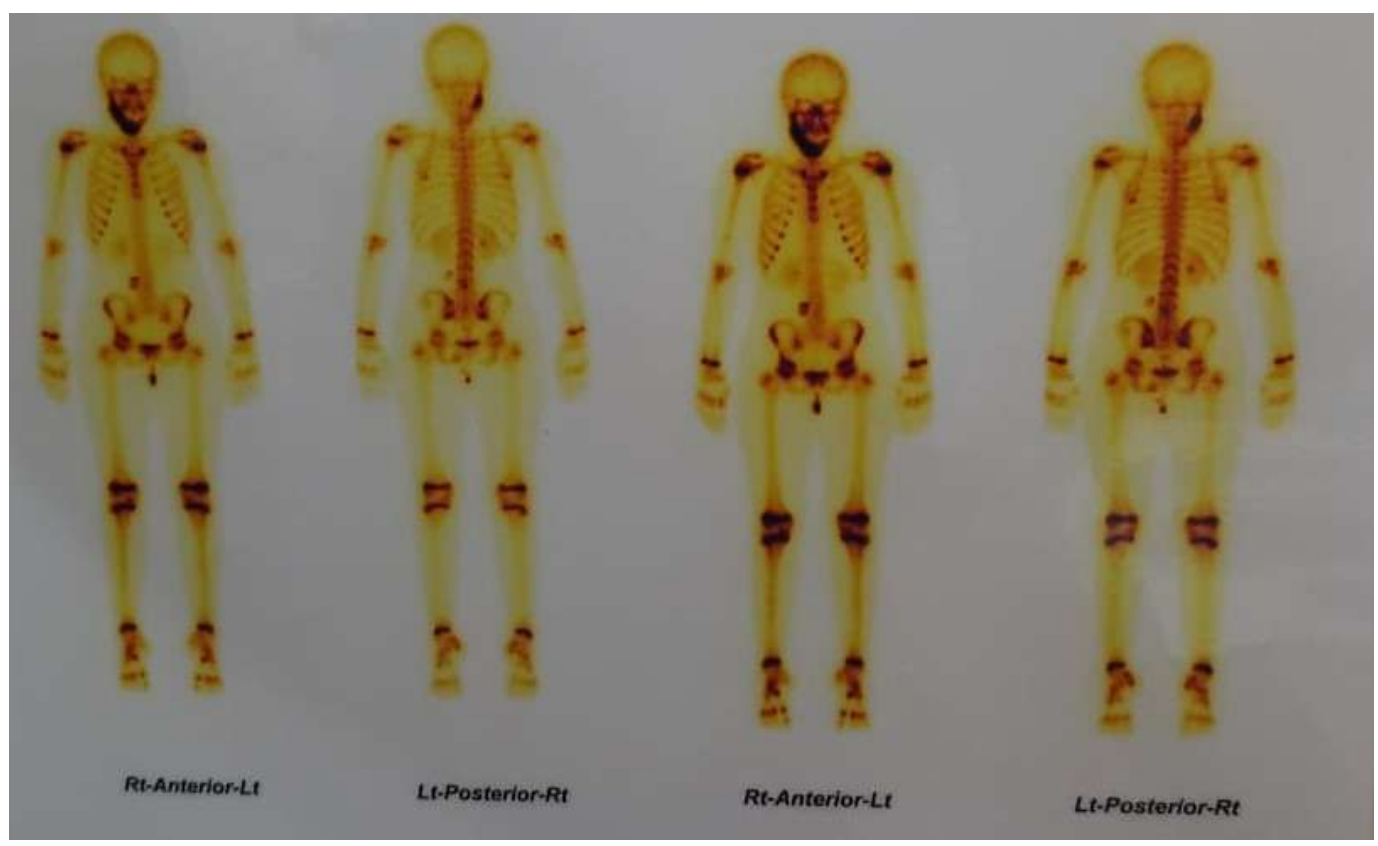

Fig-4: Bone Scintigraphy showing Lesion in Mandible (Fluorescent)

\section{DISCUSSION \& LITERTAURE REVIEW}

In 1938, Lichtenstein first coined the term "fibrous dysplasia (FD)" [3]. FD is a rare, nonmalignant condition in which normal bone and marrow are replaced by fibrous tissue and randomly distributed woven bone, usually with pain, bony deformity, and pathologic fractures [4]. In general, FD presents in three forms - monostotic, polyostotic, and polyostotic with endocrinopathies, which can be associated with hyperpigmentation and endocrinological disorders and is called McCune-Albright syndrome [5]. Common sites of skeletal involvement are the long bones, craniofacial bones, ribs, and pelvis [6]. Monostotic FD (MFD), although less serious than polyostotic FD (PFD), is of greater concern to the dentist because of the relatively high frequency of occurrence in the jaws [4]. FD is caused by somatic activating mutations of the gene GNAS in a subunit of the stimulatory $G$ protein, located at 20q13.2-13.3 [6]. The diagnosis of FD is based on physical, radiological, and histopathological examination. There are different treatment approaches including observation, medical treatment, and surgical treatment. The GNAS1 gene encodes a G-protein that stimulates the production of cAMP. The mutation results in the continuous activation of the G-protein leading to overproduction of cAMP in affected tissues. Hyperfunctioning of affected endocrine organs frequently gives rise to precocious puberty, hyperthyroidism, growth hormone excess, and overproduction of cortisol. There is increased 
proliferation of melanocytes resulting in large café-aulait spots. cAMP is thought to have effect on the differentiation of osteoblasts leading to FD [7].

Fries has described three radiological patterns in craniofacial FD $[8,9]$. The first is pagetoid or ground glass with bone expansion and alternate areas of radiodensity and radiolucency. It occurs in more than half of the patients, most of whom are older than 30 years of age and have had symptoms for an average of 15 years. The second pattern is sclerotic, with bone expansion and a homogenous radiodensity. The third type is cyst-like, usually a round or oval lesion with a sclerotic border. The sclerotic and cyst-like patterns occur in younger individuals (age below 20 years). Other features include fingerprint bone pattern, orange peel appearance, and superior displacement of mandibular canal in mandibular lesion [9]. Skeletal radiographic features include "shepherd's crook" deformity of femoral neck and "bowing deformities" in weight-bearing long bones. In most cases, the radiographic and clinical findings are sufficient to allow the practitioner to diagnose without a biopsy. Differential diagnoses with similar radiographic appearance, such as ameloblastoma, ameloblastic fibroma, ameloblastic odontoma, ameloblastic fibroodontoma, central giant cell granuloma, odontogenic cyst, ossifying fibroma, osseous dysplasia, chronic sclerosing osteomyelitis, and osteosarcoma, should be considered [10]. The density and trabecular pattern of FD lesions is variable. Early lesions may be more radiolucent than mature lesions and, in rare cases, may appear to have granular internal septa, giving the internal aspect a multilocular appearance. The abnormal trabeculae are usually shorter, thinner, irregularly shaped, and more numerous than the normal trabeculae. This creates a variable radio-opaque pattern. It may have a granular appearance ("ground-glass" appearance, resembling the small fragments of a shattered windshield), a pattern resembling the surface of an orange (peau d'orange), a wispy arrangement (cotton wool), or an amorphous, dense pattern. A distinctive characteristic is the organization of the abnormal trabeculae into a swirling pattern similar to a fingerprint [10]. Routine investigations include complete hemogram and levels of serum calcium, serum phosphorus, and serum ALP. Serum ALP is occasionally elevated, but calcium, parathyroid hormone, 25-hydroxyvitamin D, and 1,25dihydroxyvitamin D levels are normal in most cases of FD. Persons with extensive polyostotic FD may have hypophosphatemia, hyperphosphaturia, and osteomalacia. Premature secretion of pituitary follicle stimulating hormone is found. There may be an elevated basal metabolic rate [7]. The microscopic appearances are those of a hypercellular and cytologically uniform fibrous stroma within which delicate and irregularly shaped trabeculae of woven bone are deposited. The configurations of these bony trabeculae are often referred to as resembling Chinese characters [7]. Treatment of bony lesions of fibrous dysplasia includes surgical and nonsurgical therapies. Surgical treatment in young-aged minor cases and biopsy with minor bony osteoplasty at affected site are adequate. In more severe cases complete excision with graft reconstruction may be possible [11]

\section{CONCLUSION}

Fibrous dysplasia can manifest itself in a young age. Radiographic diagnosis along with histopathological diagnosis may be necessary to confirm diagnosis of the disease.

\section{REFERENCES}

1. Yang, H. Y., Su, B. C., Hwang, M. J., \& Lee, Y. P. (2018). Fibrous dysplasia of the anterior mandible: A rare case report. Tzu-Chi Medical Journal, 30(3), 185.

2. Nager, G. T., Kennedy, D. W., \& Kopstein, E. (1982). Fibrous dysplasia: a review of the disease and its manifestations in the temporal bone. The Annals of Otology, Rhinology \& laryngology. Supplement, 92, 1-52.

3. MacDonald-Jankowski, D. (2009). Fibrous dysplasia: a systematic review. Dentomaxillofacial Radiology, 38(4), 196-215.

4. Ogunsalu, C., Smith, N. J. D., \& Lewis, A. (1998). Fibrous dysplasia of the jaw bone: a review of 15 new cases and two cases of recurrence in Jamaica together with a case report. Australian dental journal, 43(6), 390-394.

5. Edgerton, M. T., Persing, J. A., \& Jane, J. A. (1985). The surgical treatment of fibrous dysplasia. With emphasis on recent contributions from cranio-maxillo-facial surgery. Annals of surgery, 202(4), 459.

6. DiCaprio, M. R., \& Enneking, W. F. (2005). Fibrous dysplasia: pathophysiology, evaluation, and treatment. JBJS, 87(8), 1848-1864.

7. Agarwal, M. M., Balaji, N., Sumathi, M. K., Sunitha, J. D., Dawar, G., \& Rallan, N. S. (2014). Fibrous dysplasia: A review. TMU J Dent. 1:2529.

8. FREIS, J. (1957). The roentgen features of fibrous dysplasis of the skull and facial bones, a critical analysis of thirty-nine pathologically proved cases. Am J Roentgenol, 77, 71-88.

9. Gursoy, B., Arslan, A., Altundal, H., \& Olgac, V. (2004). Fibrous dysplasia of the mandible: A case report. Oral Health Dent Manag, 3, 185.

10. Canýtezer, G., Gunduz, K., Ozden, B., \& Kose, H. I. (2012). Monostotic fibrous dysplasia: A case report. Dentistry. 3:1-4.

11. Moore, A. T., Buncic, J. R., \& Munro, I. R. (1985). Fibrous dysplasia of the orbit in childhood: clinical features and management. Ophthalmology, 92(1), 12-20. 